\title{
Intramedullary spinal cord and leptomeningeal metastases presenting as cauda equina syndrome in a patient with melanoma
}

\author{
Stephanie Lindauer, MD, and Michelle Janania Martinez, MD
}

Division of Hematology-Oncology, Department of Internal Medicine, University of Texas Health Science Center at San Antonio, and Cancer Therapy and Research Center, San Antonio, Texas

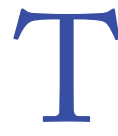

The incidence of malignant melanoma has been rising in the United States, especially among non-Hispanic white men and women. Death rates have increased for those aged 65 years or older, and incidence rates have increased for all age groups. ${ }^{1}$ It is a serious public health issue.

Given the unique biology of melanoma, metastatic disease can present in a variety of ways. In most cases, the lymph nodes and lungs are involved. ${ }^{2}$ The incidence of brain metastases is $10 \%-40 \%$, however the percentage may be even higher based on reported incidence of autopsy reports. ${ }^{3}$ The most common forms of metastatic melanoma to the spine are vertebral and intramedullary. ${ }^{4}$ Specifically, leptomeningeal involvement can be found in $20 \%$ of patients in clinical studies and $44 \%-70 \%$ in autopsy series of patients with central nervous system (CNS) metastatic disease. ${ }^{5}$ Despite its incidence, leptomeningeal disease (LMD) from melanoma is rarely discussed in the literature and the diagnosis may be difficult. Even rarer is the documented presentation of intramedullary spinal cord metastases, or "drop metastases." In our review of the literature, we found no published case reports to date of drop metastases from melanoma causing cauda equina syndrome.

The prognosis of patients with metastatic melanoma with brain metastases is very poor, with a median overall survival of about 4 months reported in several studies. ${ }^{7-9}$ Prognosis is even worse for patients with leptomeningeal involvement, and median survival without therapy is about 4-6 weeks. ${ }^{10} \mathrm{~A}$ combination of intrathecal and systemic chemotherapy can be used to treat LMD. ${ }^{11}$

\section{Case presentation and summary}

This is the case of a 56-year-old man with history of metastatic melanoma that had been initially diagnosed about 4 years before the current case presentation. Original sites of disease were a supraclavicular lymph node and solitary liver metastasis, both of which were resected. The patient then developed biopsy-proven lung involvement that required left and right wedge resections. Mutation testing for BRAF V600E and BRAF V600K was sent and not detected. Therefore the patient did not receive any BRAF-targeted therapies. Subsequently, recurrent metastatic disease to the brain with 2 dominant lesions in the cerebellum and the occiput as well as numerous small lesions at the gray-white matter junction was identified [Figure 1 and Figure 2].

The patient received whole-brain radiation (30 Gy in 10 fractions of $3 \mathrm{~Gy}$ each). There was no evidence of disease in his spine at that time. About 2 weeks after completing whole-brain radiation, the patient presented to the hospital with left lower extremity weakness, urinary retention, bowel incontinence, saddle anesthesia, and malaise. The symptoms had begun after he had finished whole-brain radiation and weakness progressed to the point at which he need a cane to be able to walk. A physical examination was significant for hyporreflexia, decreased strength and sensitivity on left lower extremity, saddle anesthesia, and lumbar spinal tenderness to palpation. The results of magnetic-resonance imaging (MRI) of the spine revealed multiple soft-tissue nodules extending from the conus medullaris throughout the cauda equina, consistent with intramedullary metastases, as well as concomitant lepto-

Accepted for publication August 1, 2016. Correspondence: Stephanie Lindauer, MD; Lindauers3@uthscsa.edu. Disclosures: The authors report no disclosures or conflicts of interest. JCSO 2017;15(4):e224-e227. (02017 Frontline Medical Communications. doi: 10.12788/icso.0293 


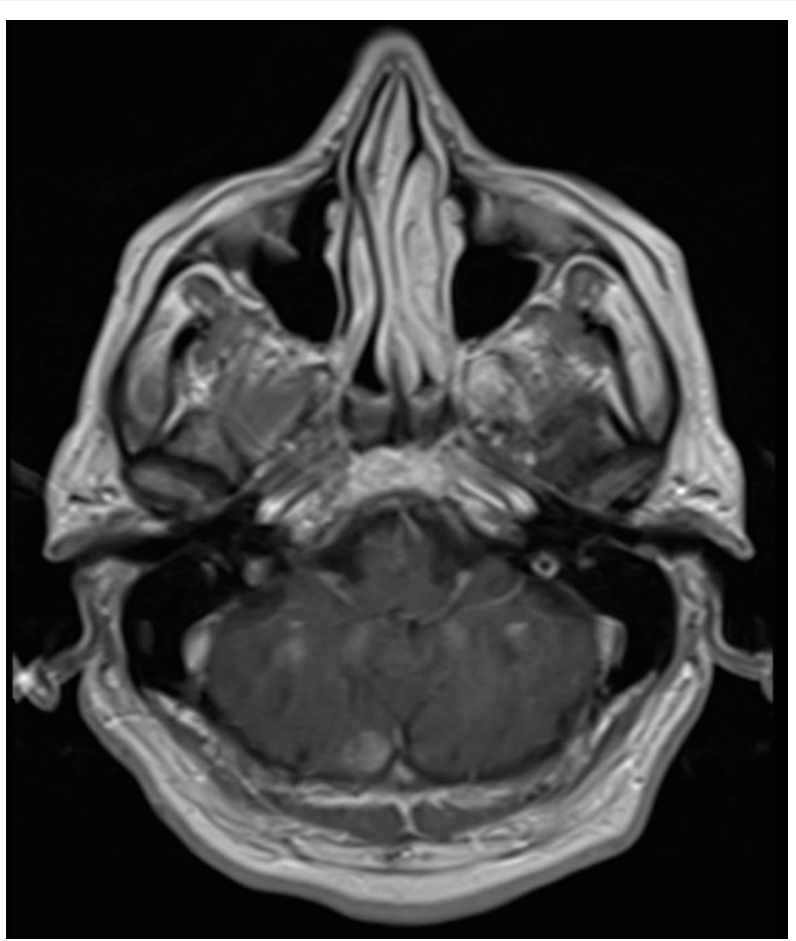

FIGURE 1 A T2-STIR sagittal view of the lumbar spine on magnetic-resonance imaging. There are multiple soft-tissue nodules within the thecal sac, extending from the conus medullaris through the cauda equina. The findings are suggestive of intramedullary metastases, or 'drop' metastases.

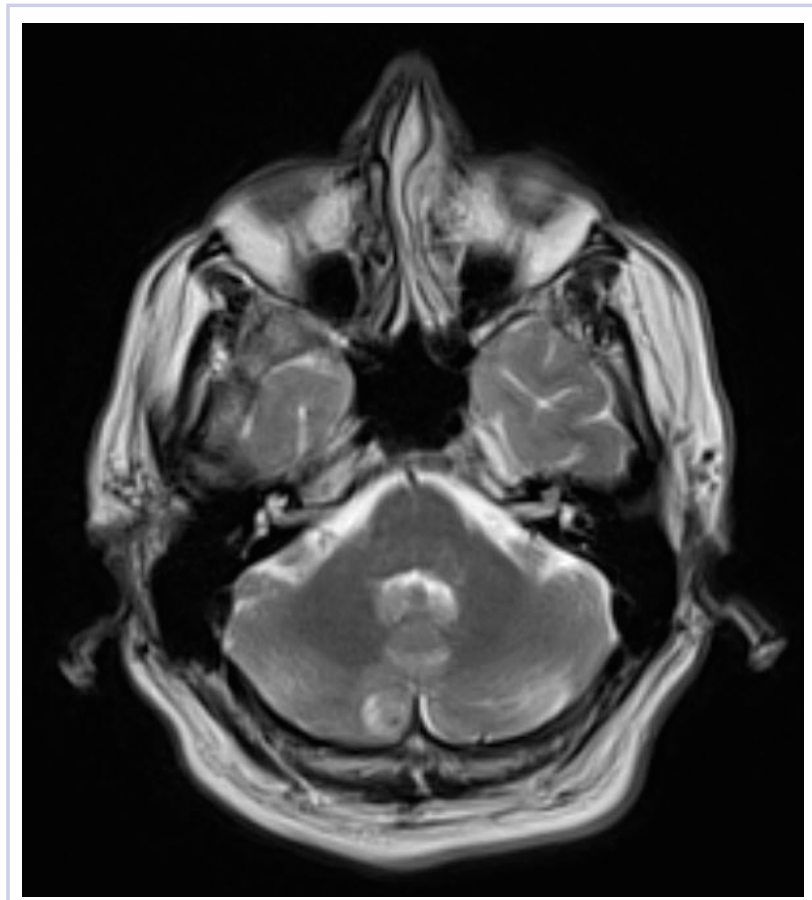

FIGURE 2 An axial T1 view of the brain on magnetic-resonance imaging. There is a $1.1-\mathrm{cm}$ diameter, enhancing lesion in the medial posterior right cerebellum consistent with metastasis. meningeal involvement [Figure 3].

The patient was started on steroids with minimal improvement in neurologic function. We consulted with our neurosurgery colleagues, but learned that no direct surgical intervention could be performed because of widespread involvement. We then proceeded with radiation, 30 Gy in 10 fractions to the lumbar spine. Intrathecal chemotherapy with methotrexate (12 $\mathrm{mg}$ twice a week) was also started, with a plan to complete 4 weeks. Shortly after starting radiation therapy and methotrexate, we observed clinical improvement in the patient, with mildly increased left lower extremity strength and increased ambulation with a physical therapist.

Cerebrospinal fluid studies (CSF) showed clearance of malignant cells after 2 treatments of intrathecal methotrexate as well as improvement in CSF chemistry parameters: the patient's protein level decreased from $1,095 \mathrm{mg} / \mathrm{dL}$ to $42 \mathrm{mg} / \mathrm{dL}(15-45 \mathrm{mg} / \mathrm{dL})$ and his glucose level increased from $3 \mathrm{mg} / \mathrm{dL}$ to $73 \mathrm{mg} / \mathrm{dL}(40-85 \mathrm{mg} / \mathrm{dL})$ However, after completing 3 weeks of intrathecal chemotherapy, the hospital course was complicated by leukopenia, thrombocytopenia, and spontaneous intracranial hemorrhage. The cytopenias were thought to be secondary to systemic effect of intrathecal methotrexate in conjunction with the radiation treatments to the spine. Intrathecal chemotherapy was held.

The patient was not a candidate for systemic immunotherapy because of his decline in performance status. $\mathrm{He}$ continued to deteriorate neurologically, and the family decided to pursue inpatient hospice. He died a week after

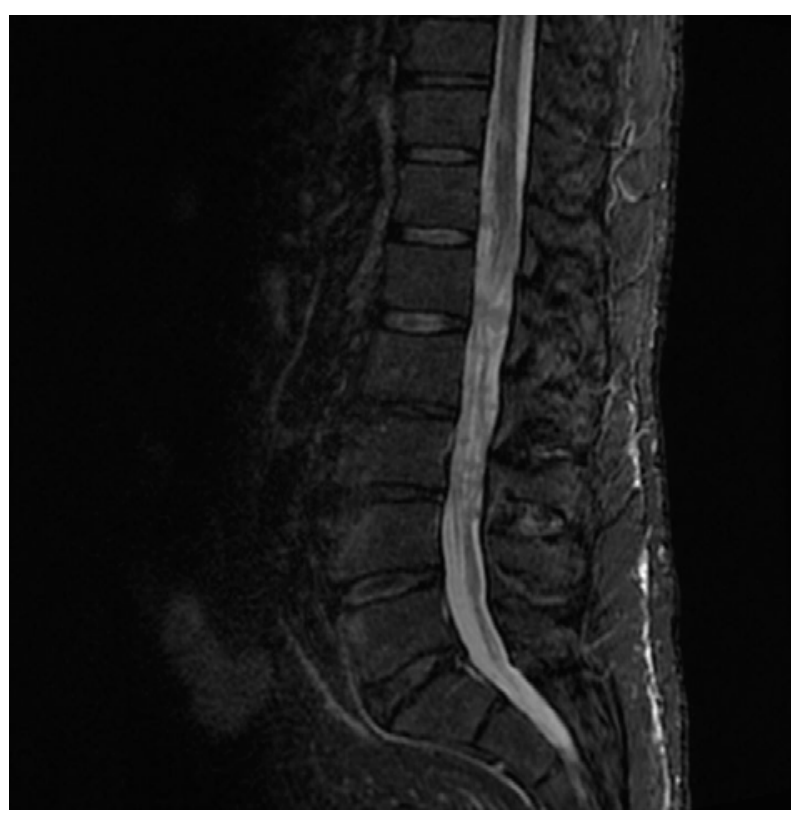

FIGURE 3 An axial T2 view of the brain on magnetic-resonance imaging. There is a $1.1-\mathrm{cm}$ diameter, enhancing lesion in the medial posterior right cerebellum consistent with metastasis. 
transfer to hospice and 5 weeks after the initial diagnosis of leptomeningeal and intramedullary metastases.

\section{Conclusions}

Although metastatic melanoma to the brain is not uncommon, leptomeningeal and intramedullary drop metastases are an infrequent presentation. Even more rare are intramedullary drop metastasis that are significant enough to cause cauda equina syndrome, as with our patient. The incidence of LMD has increased over the years and may continue to increase, likely because of the improved overall survival and a prolonged control of extracranial disease with newly approved systemic therapeutic drugs, such as molecularly targeted therapy and immunotherapy. ${ }^{12}$ Intramedullary metastases are extremely rare, but reported incidence has seemed to be increasing due to detection with MRI. Currently there are fewer than 100 case reports of intramedullary spinal cord metastasis. ${ }^{6}$ In one retrospective study, 40 patients with intramedullary metastatic disease secondary to systemic cancer were identified during 1980-1993. ${ }^{6}$ About half of those cases were from lung cancer, the second most common was breast cancer.

CNS involvement by melanoma can have debilitating complications and confers a poor prognosis. In another retrospective study, several patient characteristics were found to be associated with significantly shorter survival in patients with known brain metastases, including presence of neurologic symptoms and leptomeningeal involvement. ${ }^{3}$

Malignant cells can reach the CSF by several routes: direct extension, hematogenous, venous access, venous drainage from bone marrow and cranial and peripheral nerves. Once the tumor has reached the CSF, it can seed any portion of the nervous system that has contact with the CSF and become entangled among the cauda equine. ${ }^{13}$

Given the rarity of leptomeningeal and intramedullary involvement of melanoma, there are no standard treatment guidelines. Treatment for LMD usually consists of intrathecal and systemic chemotherapy. Commonly used intrathecal agents are methotrexate, liposomal cytarabine, and thiopeta. ${ }^{11}$ The goals of treatment are to improve or stabilize neurologic status of the patient and ideally prolong survival. The choice of agent for intrathecal chemotherapy is guided by the primary tumor, however, there is no strong evidence to choose one agent over the other. ${ }^{12,14}$ Methotrexate or cytarabine are generally recommended in the National Comprehensive Cancer Network (NCCN) guidelines. Targeted therapy toward the primary tumor is occasionally used for treatment of LMD, for example rituximab can be given intrathecally for lymphoma, ${ }^{15}$ and trastuzumab has been given intrathecally for breast cancer. ${ }^{16}$ No intrathecal targeted agents are currently available for melanoma. Administration of intrathecal chemotherapy is given via lumbar puncture or Ommaya reservoir. Induction intrathecal chemotherapy is recommended by NCCN to be given for 4-6 weeks. The schedule of administration varies based on the agent used. Most systemic chemotherapy has poor CSF penetration, which is the basis behind using chemotherapy intrathecally in these patients. ${ }^{14}$ However, novel therapies for melanoma, such as ipilimumab, have shown activity in the CNS, and it is not known if intrathecal chemotherapy will continue to play role in the management of LMD. ${ }^{17}$

Systemic therapy for metastatic melanoma has changed with the development of novel agents, which have shown better efficacy than traditional chemotherapy. The recommendation for first-line systemic therapy of metastatic unresectable melanoma is based on several factors: $B R A F$ mutation status, tempo of disease, and presence or absence of cancer-related symptoms. Immunotherapy for metastatic melanoma that is unresectable includes anti-programmed cell death protein-1 (PD-1) monotherapy (nivolumab or pembrolizumab) or combination therapy with nivolumab plus ipilimumab. Targeted therapy is preferred in cases with an identified $B R A F$ mutation. Combination therapy with dabrafenib plus trametinib or with vemurafenib plus cobimetinib is recommended. Single-agent therapy may also be used with dabrafenib or vemurafenib. ${ }^{18}$

Ipilimumab is a monoclonal antibody that blocks cytotoxic T-lymphocyte antigen-4 to potentiate an anti-tumor T-cell response that was approved in 2011 by the US Food and Drug Administration for the treatment of melanoma. A randomized, phase 3 clinical trial showed an increase in overall survival in patients with unresectable metastatic disease who had received previous treatment. ${ }^{19}$ Before that, no therapy had been shown to improve overall survival in patients with metastatic melanoma. Patients with CNS metastases were included in this study. ${ }^{19}$

The activity of ipilimumab specifically in patients with brain metastasis was further studied in a phase 2 trial that enrolled 72 patients, 1 cohort with symptomatic brain metastases and the other cohort with asymptomatic brain metastases. ${ }^{20}$ After 12 weeks of therapy, response was assessed by modified World Health Organization criteria for disease control (complete response plus partial response plus stable disease). In all, $18 \%$ of patients with asymptomatic brain metastasis achieved disease control, compared with $10 \%$ of patients with symptomatic brain metastases. When the brain alone was assessed, $24 \%$ of asymptomatic patients and $10 \%$ of symptomatic patients achieved disease control. No unexpected toxic effects occurred during the study. Anti-PD1 therapy such as nivolumab, which has shown durable responses in metastatic melanoma, has no published results specifically in patients with active brain metastases.

Of the BRAF-targeted therapy, dabrafenib and vemurafenib have also been studied in patients with brain metastases. For darafenib, 172 patients with BRAF-mutated metastatic melanoma were included in a phase 2 clinical 
trial that showed an intracranial response of 39\% in previously untreated patients and $31 \%$ in patients whose brain metastases had progressed after previous local treatment. ${ }^{21}$ Vemurafenib has also shown intracranial response in a phase 2 clinical trial. ${ }^{22}$

The role of the aforementioned therapies in patients with metastatic melanoma with CNS disease should not be overlooked because these patients are typically excluded from clinical trials. As already noted, agents such as ipilimumab and the dabrafenib-vemurafenib combination have been studied in patients with brain metastases and have shown disease control, but more studies are needed to truly assess whether there is an improvement in overall survival and whether that will change treatment guidelines. Although

\section{References}

1. Jemal A, Saraiya M, Patel P, et al. Recent trends in cutaneous melanoma incidence and death rates in the United States, 1992-2006. J Am Acad Dermatol. 2011;65(5 Suppl 1):S17.e1-S17.e11.

2. Patel JK, Didolkar MS, Pickren JW, Moore RH. Metastatic pattern of malignant melanoma: a study of 216 autopsy cases. Am J Surg. 1978;135(6):807-810.

3. Raizer J, Hwu W, Panageas K, et al. Brain and leptomeningeal metastases from cutaneous melanoma: survival outcomes based on clinical features. Neuro Oncol. 2008;10(2):199-207.

4. Sun L, Song Y, Gong Q. Easily misdiagnosed delayed metastatic intraspinal extradural melanoma of the lumbar spine: a case report and review of the literature. Oncol Lett. 2013;5(6):1799-1802.

5. Moseley R, Davies A, Bourne S, et al. Neoplastic meningitis in malignant melanoma: diagnosis with monoclonal antibiodies. J Neurol Neurosurg Psychiatry. 1989;52:991-886.

6. Schiff D, O'Neill B. Intramedullary spinal cord metastases clinical features and treatment outcome. Neurology. 1996;47(4):906-912.

7. Fife KM, Colman MH, Stevens G, et al. Determinants of outcome in melanoma patients with cerebral metastases. J Clin Oncol. 2004;22(7):1293-1300.

8. Raizer J, Hwu W, Panageas K, et al. Brain and leptomeningeal metastases from cutaneous melanoma: survival outcomes based on clinical features. Neuro Oncol. 2008;10(2):199-207.

9. Sampson JH, Carter JH Jr, Friedman AH, Seigler HF. Demographics, prognosis, and therapy in 702 patients with brain metastases from malignant melanoma. J Neurosurg. 1998;88:11-20.

10. Abernethy AP. Central nervous system tumors. In: Loprinzi C, ed. ASCO-SEP: Medical Oncology Self-evaluation Program. 4th ed. Alexandria, VA: American Society of Clinical Oncology, 2015. Page 396. Print.

11. Pape E, Desmedt E, Zairi , et al. Leptomeningeal metastasis in melanoma: a prospective clinical study of nine patients. In Vivo. 2012;26(6):1079-1086. patients with parenchymal brain metastases were included in these studies, it is not clear how patients with LMD and intramedullary spinal cord metastases, such as our patient, would be affected. It is also not clear whether intrathecal chemotherapy will continue to play a role in management of metastatic melanoma with LMD, especially if these newer agents have CNS activity in addition to controlling extracranial disease. Although rarely documented, leptomeningeal and intramedullary metastatic disease will likely become increasingly recognized as patients with cancer live longer and diagnostic studies improve. These initial studies showing intracranial disease control show compelling evidence to continue enrolling patients with active CNS disease in clinical trials.

12. Pavlidis N. The diagnostic and therapeutic management of leptomeningeal carcinomatosis. Ann Oncol. 2004;15(Suppl 4):iv285-291.

13. DeAngelis L, Posner JB. Neurologic complications of cancer. 2nd ed. New York, NY: Oxford University Press; 2008.

14. Chamberlain, M. Leptomeningeal metastasis. Curr Opin Oncol. 2010;22:627-635.

15. Chamberlain M, Johnston S, Van Horn A, Glantz MJ. Recurrent lymphomatous meningitis treated with intra-CSF rituximab and liposomal ara-C. J Neurooncol. 2009;91(3):271-277.

16. Zagouri F, Sergentanis T, Bartsch R, et al. Intrathecal administration of trastuzumab for the treatment of meningeal carcinomatosis in HER2-positive metastatic breast cancer: a systematic review and pooled analysis. Breast Cancer Res Treat. 2013;139(1):13-22.

17. Silk A, Bassetti M, West BT, Tsien C, Lao CD. Ipilimumab and radiation therapy for melanoma brain metastases. Cancer Med. 2013;2(6):899-906.

18. [Behind paywall.] National Comprehensive Cancer Network. Melanoma (version 2.2016). http://www.nccn.org/professionals/ physician_gls/pdf/melanoma.pdf. November 10,2016. Accessed February 28, 2016

19. Hodi F, O'Day S, McDermott D, et al. Improved survival with ipilimumab in patients with metastatic melanoma. NEJM. 2010;363(8):711-723.

20. Margolin K, Ernstoff M, Hamid O, et al. Ipilimumab in patients with melanoma and brain metastases: an open-label, phase 2 trial Lancet Oncol. 2012;13(5):459-465.

21. Long G, Trefzer U, Davies M, et al. Dabrafenib in patients with Val600Glu or Val600Lys BRAF-mutant melanoma metastatic to the brain (BREAK-MB): a multicentre, open-label, phase 2 trial. Lancet Oncol. 2012;13(11):1087-1095.

22. McArthur GA, Maio M, Arance A, et al. Vemurafenib in metastatic melanoma patients with brain metastases: an open-label, single-arm, phase 2, multicenter study. Ann Oncol. 2017;28(3):634-641. 